\title{
Acute headache as a presenting symptom of tacrolimus encephalopathy
}

\author{
I M Kiemeneii, F-E de Leeuw, L M P Ramos, J van Gijn
}

J Neurol Neurosurg Psychiatry 2003;74:1126-1 127

A 24 year old woman presented with a sudden excruciating headache mimicking an acute vascular event. She had undergone a lung transplantation because of cystic fibrosis and was receiving maintenance treatment with tacrolimus and prednisone. Ancillary investigation excluded vascular causes. Magnetic resonance imaging demonstrated hyperintense lesions in the infratentorial and parieto-occipital regions consistent with posterior leucencephalopathy syndrome. Both her clinical condition improved and the lesions disappeared completely after withdrawal of tacrolimus, suggesting that her condition could be explained by a tacrolimus encephalopathy.

\footnotetext{
t is well known that calcineurin inhibitors such as cyclosporine and tacrolimus, used as immunosuppressive agents in transplant patients, are neurotoxic. ${ }^{1-4}$ Mild symptoms of neurotoxicity include tremor, insomnia, nightmares, headache, vertigo, dysesthesia, photophobia, and mood disturbances. More severe symptoms are akinetic mutism, seizures, cortical blindness, focal deficits, psychosis, or decreased level of consiousness and these may ultimately lead to death. ${ }^{1}$

We present a case of tacrolimus encephalopathy that started with sudden headache, mimicking an acute vascular condition such as subarachnoid haemorrhage, deep central venous thrombosis, or dissection.
}

\section{CASE REPORT}

A 24 year old woman was admitted at our hospital on 11 June 2002 because of acute severe headache ( 9 on a scale of 0 to 10) that had started within one minute the evening before. Later that evening she became nauseous, started vomiting, and she also developed neck pain. Initially her level of consciousness was said to have been normal without focal neurological deficit. The next morning she felt lethargic and her mental functions were slow according to her family. Recent medical history revealed no head or neck trauma nor a recent infection. The patient had undergone a lung transplantation in October 2001 because of cystic fibrosis. Her current treatment consisted of tacrolimus $4 \mathrm{mg}$ and prednisone $20 \mathrm{mg}$, twice a day orally and she had recently started oral contraceptive medication (ethinylestradiol/levonorgestrel 50/125 $\mu \mathrm{g}$ ).

The initial neurological examination showed a maximum Glascow Coma Scale score although her reactions were slow; there was no focal deficit. Her blood pressure was 130/80 mm $\mathrm{Hg}$, the temperature $37.9^{\circ} \mathrm{C}$. Laboratory studies including haematology, serum electrolytes, creatinin, liver enzymes, cholesterol, magnesium, and $\mathrm{C}$ reactive protein were normal. Blood glucose concentration was increased at $12 \mathrm{mmol} / \mathrm{l}$. The tacrolimus concentration was within the therapeutic range ( 5 $\mathrm{ng} / \mathrm{ml}$ ). An initial computed tomogram of the brain showed hypodense lesions in both cerebellar hemispheres, the brain stem and the left thalamus, with slight compression of the fourth ventricle and there were no signs of subarachnoid haemorrhage. Magnetic resonance imaging, that same evening, showed hyperintense signals on fluid attenuated inversion recovery $\mathrm{T} 2$ weighted images (FLAIR) bilaterally in the cerebellum, affecting mostly the white matter, the brain stem, and corpus callosum (fig 1 A and B). Diffusion weighted imaging (DWI) revealed no acute ischaemia (fig IC and D) and magnetic resonance venography was normal. A lumbar puncture was traumatic; the CSF showed 17000 erythrocytes/ $\mathrm{mm}^{3}, 12$ white blood cells $/ \mathrm{mm}^{3}$, with a normal differentiation, CSF protein of $1.38 \mathrm{mmol} / \mathrm{l}$ and CSF glucose of $7.8 \mathrm{mmol} / \mathrm{l}$. Cultures of bacteria and viruses (JC virus, Epstein Barr virus, cytomegalovirus, toxoplasmosis) in CSF were negative. No evidence of recent infection by these viruses was found by means of PCR techniques in serum or CSF.

As we suspected that her clinical condition was explained by tacrolimus encephalopathy, the tacrolimus was withdrawn and immunosuppressive treatment was changed to cyclosporine (200 mg, twice a day) and mycophenolate mofetil ( $750 \mu \mathrm{g}$ twice a day) on the day of admission. The next few days the patient felt better; her headache disappeared and she was more alert. On day 7 of admission she again experienced severe headache, nausea, drowsiness, and blurred vision. On examination she now had a left homonymous hemianopia. Blood pressure was increased at 150/100 mm Hg. Repeated laboratory screening showed no abnormalities. The serum concentration of cyclosporine was $0.13 \mathrm{mg} / \mathrm{l}$. A second magnetic resonance scan of the brain demonstrated more marked hyperintense lesions in the cerebellum on $\mathrm{T} 2$ weighted images and new hyperintense lesions in the right parieto-occipital lobe (figlE-H). Subsequently, cyclosporine was also withdrawn and a calcineurin inhibitor sparing regimen was started by adding sirolimus ( $5 \mathrm{mg}$ once a day) and increasing the dose of mycophenolate mofetil to $1 \mathrm{mg}$ twice a day. After withdrawal of cyclosporine the signs and symptoms gradually cleared in the course of one week; the homonymous hemianopia almost disappeared. The patient could be discharged in good clinical condition 15 days after admission. A third MRI scan showed almost no abnormalities anymore and the fourth ventricle had regained its normal shape (fig II and $\mathrm{J})$. The patient had no residual symptoms when she visited our outpatient clinic six weeks after discharge.

\section{DISCUSSION}

Tacrolimus encephalopathy and cyclosporine encephalopathy are a form of reversible posterior leucencephalopathy (PLE). ${ }^{2-4}$ This syndrome also includes hypertensive encephalopathy and the encephalopathy seen in eclampsia. ${ }^{2-4}$ The pathogenesis of PLE is largely unknown. The lesions of PLE can best be visualised by MRI with fluid attenuated inversion recovery. ${ }^{5}$ Diffusion weighted images and apparent diffusion coefficient maps in the case of PLE are mostly normal or show areas with increased diffusion, suggesting neurotoxicity rather than acute ischaemia (low diffusion). It has been postulated that tacrolimus and cyclosporine have direct toxic 


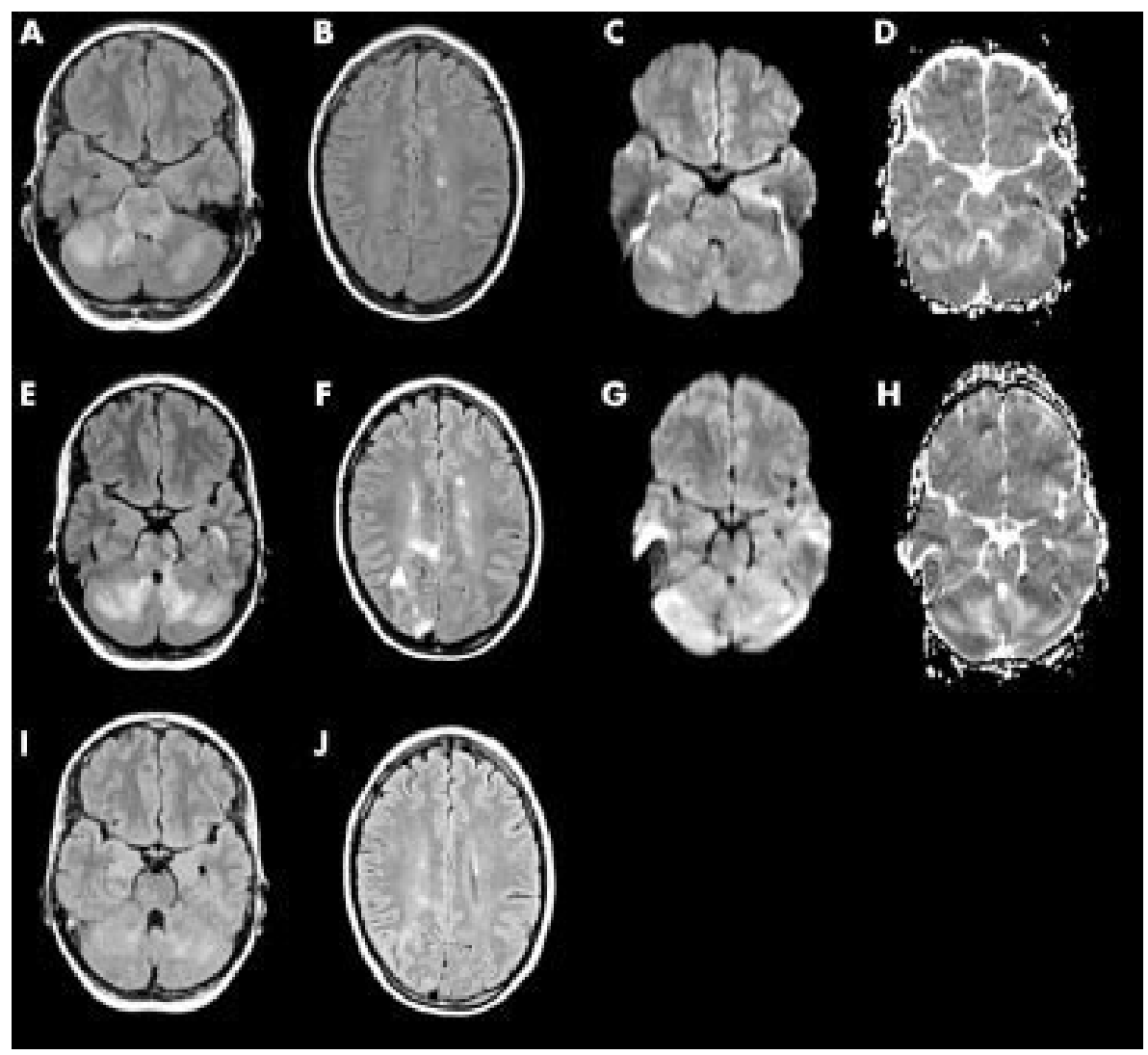

Figure 1 (A) and (B) MRI-FLAIR on admission; axial sections show hyperintense lesions in both cerebellar hemispheres, pons $(\mathrm{A})$ and in left centrum semi ovale (B). (C) and (D) MRI-DWI and apparent diffusion coefficient (ADC) map on admission show some increased diffusion in the right cerebellar hemisphere. No signs of acute ischaemia. (E) and (F) MRI-FLAIR on day 7 ; more hyperintense lesions in cerebellum (E) and new clinical relevant lesions in right parieto-occipital lobe (F). (G) and $(H)$ MRI-DWI and ADC map show increased diffusion in right cerebellar hemisphere. (I) and (J) MRI-FLAIR on day 15; normal shape of fourth ventricle and almost complete resolution of hyperintense lesions in cerebellum (I) and right parieto-occipital lobe (J).

effects on vascular endothelial cells in the nervous system, resulting in the release of endothelin, prostacyclin, and thromboxane, which in turn causes uncontrolled vasoconstriction and vasospasm in cerebral vessels. This impairment of cerebral blood flow may lead to white matter oedema and reversible ischaemia. ${ }^{4}$ The reason for the susceptibility of the posterior part of the brain to these disturbances in cerebral blood flow is not clear but a possible factor is the paucity of perivascular sympathetic innervation, an important control mechanism of cerebral blood flow. The lack of sympathetic tone may result in less protection against perfusion changes in the posterior part of the brain. The alternation of vasoconstriction and dilatation may also explain the sudden and severe headache in our patient. This is supported by another study that found segmental intracerebral arterial vasoconstriction and vasospasm in acute idiopathic headache. ${ }^{6}$ Theoretically, the acute headache could also be explained by a demyelinating lesion in the periaquaductal grey matter, as this has previously been described as a presenting symptom of multiple sclerosis. ${ }^{7}$ However, a normal cerebrospinal fluid analysis, complete resolution of lesions on MRI, and the absence of a lesion in the periaquaductal grey matter excluded this explanation for the symptoms in our patient. Generally symptoms of PLE present in subacute fashion and gradually progress, except in case of sudden onset seizures. Headache is a common symptom of PLE and mostly described as mild. The presentation of our patient with an acute severe headache in tacrolimus encephalopathy is very unusual. Ancillary investigations excluded vascular causes. That the headache subsided after tacrolimus and subsequently cyclosporine were withdrawn suggests a causal relation with the use of these drugs. It is important to recognise tacrolimus encephalopathy in addition to the more usual manifestations of PLE because irreversible brain damage or even death may occur if adequate actions are not undertaken.

\section{Authors' affiliations}

I M Kiemeneii, Department of Neurology, University Medical Centre Utrecht, St Elisabeth Medical Centre Tilburg, Netherlands

F-E de Leeuw, J van Gijn, Department of Neurology, University Medical Centre Utrecht

L M P Ramos, Department of Radiology, University Medical Centre Utrecht

\section{Competing interests: none declared.}

Correspondence to: Dr F-E de Leeuw, University Medical Centre St Radboud, Nijmegen, PO Box 9101, 6500IIB Nijmegen,

The Netherlands; hdeleeuw@neuro.umcn.nl

Received 5 November 2002

Revised 24 January 2003

Accepted 11 February 2003

\section{REFERENCES}

1 Bargallo N, Burrel M, Berenguer J, et al. Cortical laminar necrosis caused by immunosuppressive therapy and chemotherapy. Am J Neuroradiol 2000;21:479-84.

2 Hinchey J, Chaves C, Appignani B, et al. A reversible posterior leukencephalopathy syndrome. N Engl J Med 1996;334:494-500.

3 Gijtenbeek JMM, van den Bent M, Vecht ChJ. Cyclosporine neurotoxicity: a review. J Neurol 1999;246:339-46.

4 Bechstein WO. Neurotoxicity of calcineurin inhibitors:impact and clinical management. Transpl Int 2000;13:313-26.

5 Casey SO, Sampaio RC, Michel E, et al. Posterior reversible encephalopathy syndrome: utility of fluid-attenuated inversion recovery MR imaging in the detection of cortical and subcortical lesions. Am J Neuroradiol 2000;21:1199-206.

6 Slivka A, Philbrook B. Clinical and angiographic features of thunderclap headache. Headache 1995;35:1-6.

7 Haas DC, Kent PF, Friedman DI. Headache caused by a single lesion of multiple sclerosis in the periaquaductal gray area. Headache 1993;33:452-5. 